\title{
An unexpected cause of right ventricular failure - an intruder in the right ventricular outflow tract
}

\section{DDubravka Šušnjar* Dandra Jakšić Jurinjak', \\ (DIlko Vuksanović', DMario Udovičić1, (D) Josip Vincelj², (DIgor Rudež ${ }^{1,3}$}

'University Hospital Dubrava Zagreb, Zagreb, Croatia 2University Josip Juraj Strossmayer Osijek, Faculty of Medicine, Osijek, Croatia

${ }^{3}$ University of Zagreb School of Medicine, Zagreb, Croatia
KEYWORDS: right ventricular heart failure, foreign body, pulmonary valve insufficiency. CITATION: Cardiol Croat. 2019;14(3-4):76-7. | https://doi.org/10.15836/ccar2019.76

*ADDRESS FOR CORRESPONDENCE: Dubravka Šušnjar, Klinička bolnica Dubrava, Avenija Gojka Šuška 6, HR-10000, Zagreb, Croatia. / Phone: +385-99-290-4019 / E-mail: dubravka.susnjar@gmail.com

ORCID: Dubravka Šušnjar, https://orcid.org/0000-0002-9644-9739 • Sandra Jakšić Jurinjak, https://orcid.org/0000-0002-7349-6137 Ilko Vuksanović, https://orcid.org/0000-0002-4462-8647 • Mario Udovičić, https://orcid.org/0000-0001-9912-2179 Josip Vincelj, https://orcid.org/0000-0003-0064-9128 • Igor Rudež, https://orcid.org/0000-0002-7735-6721

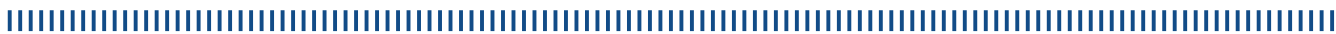

Case report: 56-year-old male patient was admitted due to fever of unknown origin. Upon admission the patient was in bad general condition with high values of inflammatory markers in laboratory results and signs of right side heart failure. 12-lead ECG showed nonspecific conduction disorders. No signs of systemic disease have been found with extensive internal and diagnostic treatment. Scintigraphy with labeled leukocytes, as attempt to find origin of infection did not show any pathological accumulation. Coronarography excludes atherosclerotic changes in epicardial vessels. Transthoracic (TTE) and transesophageal (TEE) echocardiography described a visible hyperechogenic formation in a right ventricular outflow tract (RVOT), oriented towards pulmonic valve, $1.6 \mathrm{~cm}$ long and $0.3 \mathrm{~cm}$ wide (Figure 1). Right ventricle (RV) showed milder reduced systolic function, with signs of right-side congestion. There was moderate pulmonary valve regurgitation (PR 2+), and mild tricuspid regurgitation
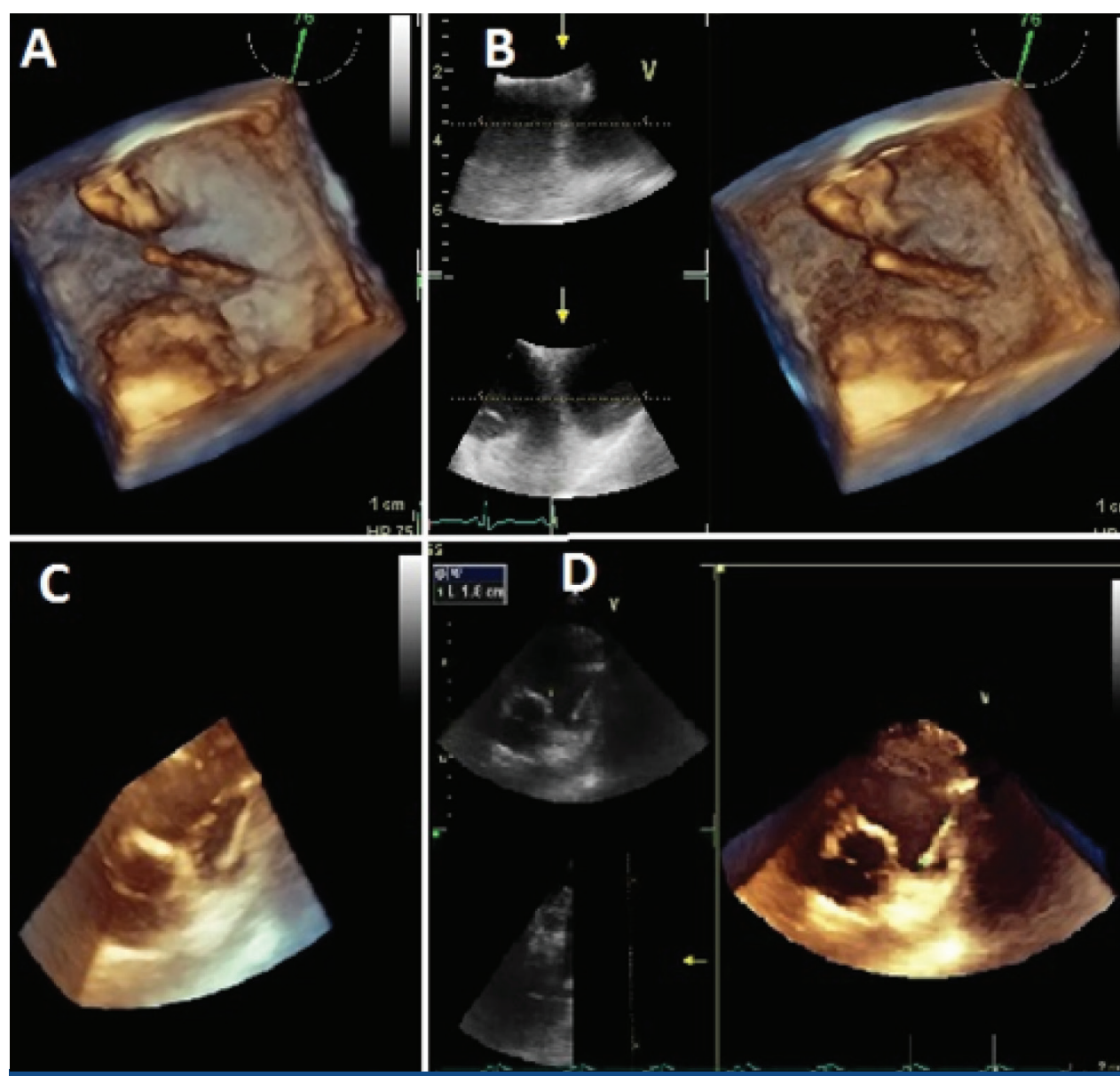

FIGURE 1. A, B 3D-Transoesophageal echocardiography of foreign body in right ventricular outflow tract. B, C 3D-Transthoracic echocardiography revealing foreign body in right outflow tract. $\square$ Cardiologia Croatica 2019:14(3-4):76.
10. hrvatski dvogodišnji ehokardiografski kongres s međunarodnim sudjelovanjem $10^{\text {th }}$ Croatian Biennial Echocardiography Congress with International Participation Poreč, 16. do 18.5.2019. 
with estimation of right ventricular systolic pressure of $27 \mathrm{mmHg}$. Preserved left ventricular fraction of $58 \%$ was observed. MSCT of thorax identified a strange metal body, resembling to sewing needle in the RV area. The patient initially refused the surgical procedure of foreign body extraction, until the clinical condition deteriorated. He was hospitalized again with fever, hem culture positive on Escherichia coli. Transthoracic echocardiography now showed a formation of $8 \mathrm{~mm}$, possibly vegetation, on the ventricular surface of the pulmonary valve with severe pulmonary insufficiency, severe tricuspid regurgitation and moderate right-side heart failure. Diagnosis of pulmonary valve endocarditis has been established. The patient was successfully operated, the bioprosthetic pulmonary valve was implanted combined with tricuspid valve repair and the foreign body was removed from the right ventricle. Postoperative recovery went well, and control echocardiography showed a good function of the bioprosthetic pulmonary valve and tricuspid valve repair. Foreign body was a sewing needle, but the patient could not remember how did it get there.

Conclusion: Isolated right ventricular heart failure can be caused by pulmonary valve insufficiency ${ }^{1,2}$, as in our case by foreign body in RVOT finally complicated by endocarditis of pulmonary valve. 